\title{
Dimensiones de la cresta ósea vestibular en incisivos maxilares con indicación de implantes inmediatos. Un estudio transversal y sus implicaciones en el plan de tratamiento.
}

\author{
Dimensions of facial bone ridge in maxillary incisors with \\ immediate implants indication. A cross-sectional study and \\ treatment plan implications.
}

\author{
Alejandro Sierra-Rebolledo ${ }^{1 *}$, Rogelio Jimenez-Tortolero .
}

\author{
1. Area de Cirugía, Departamento de \\ Estomatoquirúrgica, Facultad de Odontología, \\ Universidad de Carabobo. Valencia, Venezuela. \\ * Correspondencia Autor: Alejandro Sierra- \\ Rebolledo | Departamento de Estomatoquirúrgica, \\ Area de Cirugía, Pabellón 9, Facultad de \\ Odontología, Campus de Barbula, 2005, Valencia, \\ Venezuela|E-mail: asierra4@uc.edu.ve \\ Trabajo recibido el 17/04/2020. \\ Aprobado para su publicación el 14/05/2020
}

\section{RESUMEN}

Objetivo: Determinar las dimensiones de la cresta ósea vestibular de los incisivos maxilares con indicación de implantación inmediata. Pacientes y método: Un estudio transversal fue realizado en pacientes con necesidad de colocación de implantes inmediatos unitarios en la zona incisiva superior, durante el periodo de Enero-2015 a Diciembre-2017. Cortes tomográficos sagítales fueron utilizados para determinar la altura y el grosor de la cresta ósea alveolar vestibular. El punto de medición del grosor fue localizado a $4 \mathrm{~mm}$ apical a la linea amelocementaria. Un análisis T-student, fue utilizado para comparar las variables según la edad, el género y el grupo dentario, con un intervalo de confianza de 95\%. Resultados: 298 imágenes fueron incluidas en la evaluaciónn. El promedio de altura fue 10,68 mm, no hubo diferencias al comparar los grupos. El grosor promedio fue de $0,73 \mathrm{~mm}$, diferencias de grosor, estadísticamente significativas, fueron observadas al comparar la edad y el género, no así en el grupo dentarlo. Conclusiones: La altura del hueso alveolar vestibular de incisivos superiores es suficiente para colocar implantes inmediatos dentro de un marco óseo. No obstante, el grosor observado, se traduciría en la necesidad de complementar la implantación con técnicas de regeneraciónn tisular guiada.

\section{PALABRAS CLAVE}

Proceso alveolar; Implantes dentales; Regeneración ósea; Envejecimiento biológico; Interpretación imágenes; Hueso Maxilar.

Int. J. Inter. Dent Vol. 13(2); 71-75, 2020.

\section{ABSTRACT}

Objective : Determine the dimensions of the facial bone ridge of the maxillary incisors with indication of immediate implantation. Patients and method: A cross-sectional study was carried out in patients in need of single immediate implant placement in the upper incisor area, during the period from January-2015 to December-2017. Sagittal tomographic sections were used to determine the height and thickness of the vestibular alveolar bone ridge. The thickness measurement point was located $4 \mathrm{~mm}$ apical to the amelocementary junction. A T-student analysis was used to compare the variables according to age, gender, and dental group, with a 95\% confidence interval. Results: 298 images were included in the evaluation. The mean height was $10.68 \mathrm{~mm}$, there were no differences when comparing the groups. The mean thickness was $0.73 \mathrm{~mm}$, statistically significant differences in thickness were observed when comparing age and gender, but not in the dental group. Conclusions: The height of the vestibular alveolar bone of the upper incisors could be sufficient to place immediate implants within a bone framework. However, the thickness observed would result in the need to complement the implantation with guided tissue regeneration techniques.

\section{KEY WORDS}

Alveolar process; Dental implants; Bone regeneration; Biological aging; Image interpretation; Maxillary bone.

Int. J. Inter. Dent Vol. 13(2); 71-75, 2020. 


\section{INTRODUCCIÓN}

La pérdida de unidades dentarias en el sector anterior maxilar produce alteraciones estructurales capaces de alterar la calidad de vida del individuo dentro de la sociedad. Producto de esta ausencia una disminución de las dimensiones óseas se observa través del tiempo debido a la pérdida de las estructuras del sostén del diente, la falta de estímulos biomecánicos y propioceptivos en el hueso y la sobrecarga funcional resultante del uso de prótesis removibles ${ }^{(1,2)}$. Desde sus comienzos, los implantes dentales, han demostrado ser predecibles, en cuanto a la conservación de las dimensiones del hueso alveolar, especialmente cuando son comparados con las prótesis dentales convencionales ${ }^{(3-5)}$. Recientemente, se ha propuesto a la implantación inmediata como una alternativa eficaz para la conservación de las estructuras óseas y tejidos blandos periimplantarios ${ }^{(6-8)}$

Una prótesis implanto soportada estética debe estar en armonía con las estructuras faciales y peribucales del paciente ${ }^{(9)}$. Es por ello que la presencia de un hueso alveolar óptimo en cuanto a altura y espesor, será un factor determinante no solo durante la planificación, también en la preservación de la posición de los tejidos blandos indispensables para el éxito estético ${ }^{(10)}$.

Durante la colocación del implante, un grosor de la pared vestibular $>2 \mathrm{~mm}$ será indispensable para asegurar un buen soporte de los tejidos blandos y prevenir la resorción ósea ${ }^{(11-13)}$. Sin embargo, una pérdida progresiva del hueso crestal vestibular acompañado de cambios en la altura del margen gingival se han observado durante la evolución de los implantes, especialmente en el sector anterior del maxilar ${ }^{(14,15)}$.

Desde su primera aplicación, la tomografía computarizada de haz cónico (CBCT) se ha convertido en una herramienta eficaz para la evaluación del hueso y la planificación de los implantes en una correcta posición ${ }^{(16)}$. Braut et al $^{(17)}$; analizaron el espesor de la pared del hueso vestibular en los dientes maxilares anteriores utilizando imágenes por $\mathrm{CBCT}$, sus resultados muestran que la mayoría de casos el grosor de la pared vestibular no supera $1 \mathrm{~mm}$, adicionalmente en un $90 \%$ de los pacientes, la pared ósea vestibular en el área de la cresta de los dientes en el maxilar anterior faltaba o era fina. Miyamoto y Obama ${ }^{(16)}$, evaluaron la influencia del espesor del hueso alveolar vestibular en la pérdida ósea vertical de las recesiones gingivales postoperatorias alrededor de los implantes maxilares anteriores. Los autores concluyen que el espesor del hueso vestibular, medido por CBCT, constituyó un indicador eficaz para evaluar la recesión gingival en la región.

Estudios recientes han determinado una menor pérdida de hueso marginal crestal al implante cuando la profundidad del implante se ubica a 1 o $2 \mathrm{~mm}$ subcrestal, lo que sería determinante para obtener un resultado estético predecible ${ }^{(13,18)}$. Esto nos indicaría la necesidad de conocer la anatomía del hueso alveolar previamente a la extracción dentaria, con la finalidad de poder realizar una correcta planificación de la posición final del implante, permitiendo ofrecer rehabilitaciones estéticas predecibles y duraderas. De allí surge el objetivo de la presente investigación que fue determinar mediante la evaluación de imágenes de tomográficas las dimensiones del hueso alveolar de los incisivos superiores cuantificando la altura y el grosor de la cortical vestibular tomando en cuenta además variables como género y edad.

\section{MATERIALES Y MÉTODO}

Un estudio transversal comparativo con diseño no experimental, fue realizado en un grupo de pacientes que acudieron a dos centros privados en las ciudades de Valencia y Maracay, Venezuela, durante el periodo de tiempo comprendido entre enero de 2015 y diciembre de 2017, con necesidad de colocación de uno o mas implantes inmediatos unitarios en el sector anterior maxilar. Una muestra no probabilística de tipo intencional, que incluía a todos los pacientes mayores de 18 años de edad, sin historia de diabetes, trastorno del metabolismo óseo, o antecedentes de haber sido tratado con radioterapia de cabeza y cuello o con bifosfonatos orales o endovenoso, fueron incluidos para la evaluación. Los casos con antecedente de tratamiento ortodóntico o enfermedad periodontal fueron excluidos de la evaluación, y de acuerdo a la declaración de Helsinsky (2018) y siguiendo los lineamientos de STROBE, todos los pacientes firmaron un consentimiento informado para incluirse en el estudio ${ }^{(19)}$.

Las imágenes obtenidas de los cortes sagitales realizados a los incisivos central o lateral, provenientes de un CTCB NewTom VGI (NewTom, Verona, Italia), con un FOV de 8x8 y en modo de alta resolución fueron utilizados. La aplicación para visión de imágenes NNT viewer versión 5.6.0, fue utilizada para realizar cortes sagitales al eje longitudinal de cada diente con un espesor de $0,3 \mathrm{~mm}$.

Los cortes de los pacientes fueron agrupados según la edad. Basados en un estudio previo donde se observó una disminución de la dimensiones del hueso luego de 45 años $^{(20)}$, los grupos se determinaron en: Grupo 1 (menores de 45 años) y Grupo 2 (mayores de 45 años). Adicionalmente el género (Masculino y Femenino) y el tipo de diente del grupo incisivo, incisivo central (IC) o incisivo lateral (IL) fueron utilizadas para realizar una comparación de la muestra y completar la evaluación.

Medición de la Altura de la Cresta Ósea Vestibular (ACV) y el Grosor de la Cresta Ósea Vestibular (GCV):

La distancia en milímetros desde el punto donde coincide la proyección hacia vestibular de una línea perpendicular trazada en el ápice del diente con la cortical ósea vestibular hasta el punto más crestal de la misma cortical ósea vestibular fue cuantificado para determinar la altura del hueso.

En la actualidad, la profundidad ideal de los implantes Inmediatos ha sido establecida entre 1 a $2 \mathrm{~mm}$ subcrestal. Por tal motivo, un punto localizado a $4 \mathrm{~mm}$ apical a la línea amelocementaria del diente, fue determinado por los investigadores para medir los valores en milímetros correspondientes al grosor del hueso en sentido vestíbulo palatino, asegurándose así hacer la medición en un punto teórico de profundidad del implante. En aquellos casos donde no se observó hueso en ese punto se registró con el valor cero. (Figura. 1)

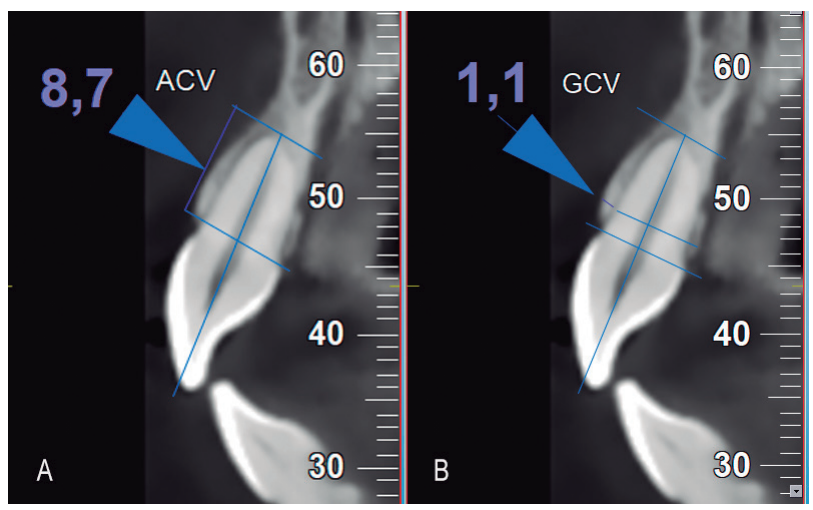

Figura 1. Corte sagital de incisivo central donde se observa el método de medición de las variables: A.medición de la altura de la cresta ósea vestibular desde una línea proyectada hacia vestibular del ápice dentarlo hasta el punto más alto de hueso; B. medición del grosor óseo vestibular a $4 \mathrm{~mm}$ apical a la línea.

\section{Análisis de lo datos.}

Los datos fueron registrados mediante el paquete estadístico IBM SPSS 22.0. La prueba de normalidad Kolmogorov-Smirnov y un análisis comparativo mediante T-student fue realizado para ambas variables altura y grosor, con un intervalo de confianza de $95 \%(p<.05)$.

\section{RESULTADOS}

Un total de 298 cortes tomográficos provenientes de la misma cantidad de pacientes pacientes, $158(52,8 \%)$ mujeres y $141(47,2 \%)$ hombres, con edad promedio de 42,36 $\pm 17,72$ años con un rango de 18-76 años, fueron incluidos en la evaluación. De la totalidad de cortes $149(49,8 \%)$ correspondían a incisivos centrales y unos 150 (49,5\%) cortes pertenecían a incisivos laterales. No se realizó distinción entre maxilares, la distribución de la muestra por grupos se muestra en la tabla 1.

Tabla 1: Distribución en valores absolutos y relativos de la muestra por grupo asignado, género y tipo de diente.

\begin{tabular}{l|c|c}
\hline & $\mathrm{N}$ & $\%$ \\
\hline Grupos & & \\
\hline 1 (Menores de 45 años) & 158 & 53,0 \\
2 (Mayores de 45 años) & 140 & 47,0 \\
\hline Genero & & \\
\hline Masculino & 140 & 47,0 \\
Femenino & 158 & 53,0 \\
\hline Grupo Dentario & 149 & 50,0 \\
\hline Incisivo Central & 149 & 50,0 \\
Incisivo Lateral & 298 & 100 \\
\hline \multicolumn{2}{c}{ Total } & \\
\hline
\end{tabular}


Tabla 2: Valores promedios, mediana y rango de la altura de la cresta ósea vestibular en milímetros según las variables de agrupamiento.

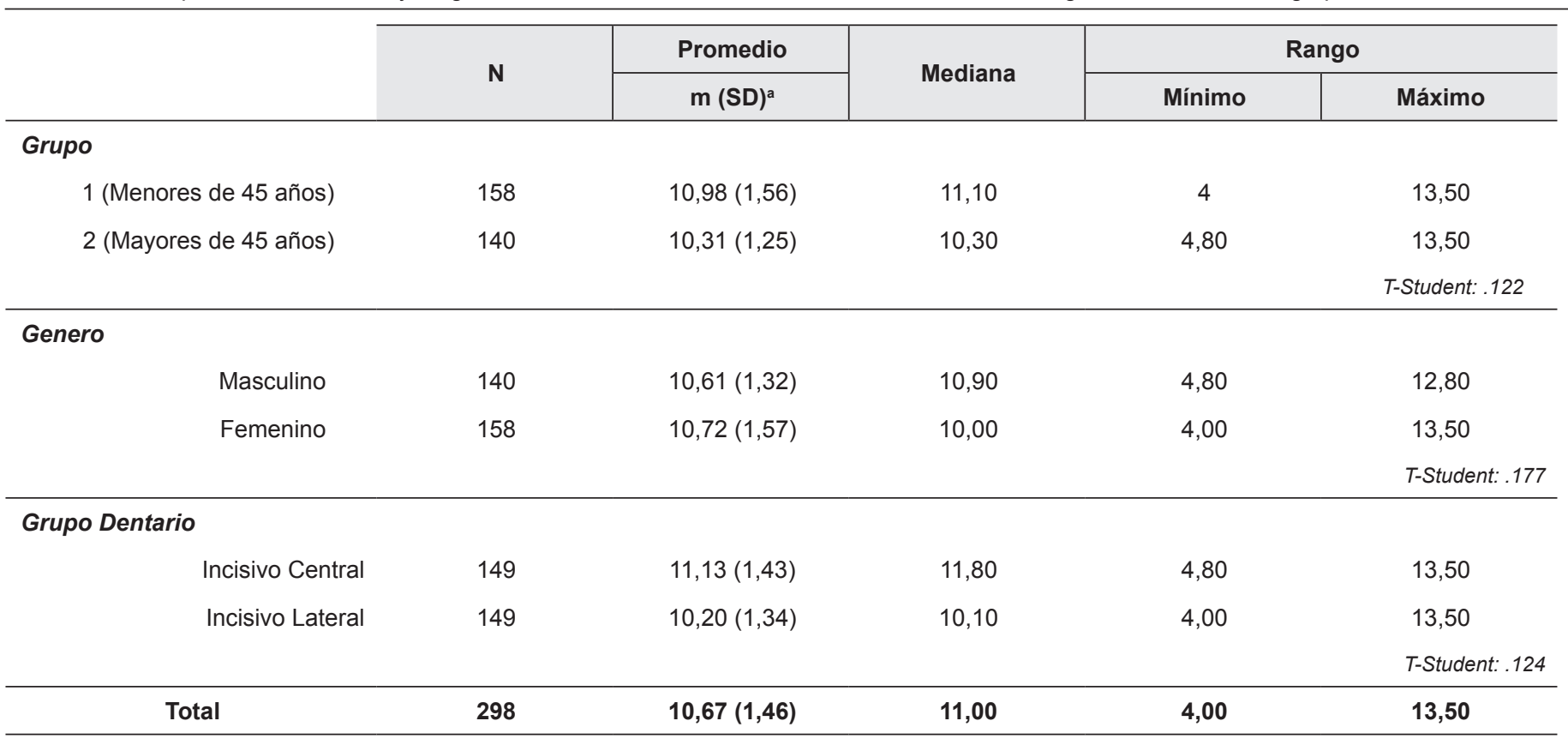

*Valores significativos $p<.05$

a. medias y Desviación estándar

Tabla 3: Valores promedios, mediana y rango del grosor de la cresta ósea vestibular en milímetros según las variables de agrupamiento.

\begin{tabular}{|c|c|c|c|c|c|c|}
\hline & & \multirow{2}{*}{$\mathbf{N}$} & \multirow{2}{*}{$\begin{array}{c}\text { Promedio } \\
\mathrm{m}(\mathrm{SD})^{\mathrm{a}}\end{array}$} & \multirow{2}{*}{ Mediana } & \multicolumn{2}{|c|}{ Rango } \\
\hline & & & & & Mínimo & Máximo \\
\hline \multicolumn{7}{|c|}{ Grupo } \\
\hline & 1 (Menores de 45 años) & 158 & $0,99(0,33)$ & 1,00 & 0 & 2,70 \\
\hline & 2 (Mayores de 45 años) & 140 & $0,43(0,59)$ & 0,00 & 0 & 2,30 \\
\hline & & & & & & ${ }^{*} T$-Student: .000 \\
\hline \multicolumn{7}{|c|}{ Genero } \\
\hline & Masculino & 140 & $0,59(0,53)$ & 0,90 & 0,00 & 2,00 \\
\hline & Femenino & 158 & $0,85(0,54)$ & 0,90 & 0,00 & 2,70 \\
\hline & & & & & & ${ }^{*}$ T-Student: .000 \\
\hline \multicolumn{7}{|c|}{ Grupo Dentario } \\
\hline & Incisivo Central & 149 & $0,71(0,53)$ & 1,00 & 0,00 & 2,20 \\
\hline & Incisivo Lateral & 149 & $0,75(0,56)$ & 1,10 & 0,00 & 2,70 \\
\hline & & & & & & T-Student: .152 \\
\hline Total & & 298 & $0,73(0,55)$ & 0,90 & 4,00 & 13,50 \\
\hline
\end{tabular}

* Valores significativos $p<.05$

a. medias y Desviación estándar

La prueba Kolmogorov-Smirnov comprobó la normalidad de la muestra en cuanto a las variables Edad, ACV y GCV.

Altura de la cresta Vestibular (ACV):

El promedio de altura de la cresta ósea vestibular fue 10,68 $\pm 1,48$ $\mathrm{mm}$, observando mayores valores de alturas en el género femenino y en el grupo 1. El grupo IC fue el grupo dentario que presentó el mayor promedio de altura de hueso de la cresta vestibular con 11,13 $\pm 1,43 \mathrm{~mm}$. no se observaron diferencias significativas al comparar los grupos entre sí. (Tabla 2)

Grosor de la Cresta Vestibular (GCV).

El promedio de grosor de la cresta vestibular observado en todas las imágenes evaluadas fue de $0,73 \pm 0,55 \mathrm{~mm}$. Nuevamente, los mayores espesores de hueso se encontraron en el grupo 1 con un promedio de 1,00 $\pm 0,34 \mathrm{~mm}$, comparados con los $0,43 \pm 0,57 \mathrm{~mm}$ observados en el grupo 2 , este valor resultó ser estadísticamente significativo. Paralelamente, resalta el hecho de que la mediana de este último grupo de mayores de 45 años fue $0,00 \mathrm{~mm}$, denotando una ausencia de hueso a este nivel en la mayoría de los pacientes evaluados.

En cuanto al género, mayores valores de grosor fueron encontrado en el grupo femenino $0,85 \pm 0,54 \mathrm{~mm}$ comparados con los valores obtenidos en el género masculino, esta diferencia de valores resultaron ser estadísticamente significativas $(p=.000)$. A diferencia de la altura, un mayor espesor óseo se observa en el grupo de incisivos laterales comparado con los incisivos centrales. (Tabla 3)

El diseño del estudio determinó que la medición del grosor se debía 
Tabla 4: Frecuencia de subgrupos en base al valor del grosor de la cortical ósea en milimetros separados por grupo etarios.

\begin{tabular}{|c|c|c|c|c|c|c|c|c|c|c|c|c|}
\hline Menores de 45 años & 5 & 1,7 & 0 & 0 & 131 & 44,0 & 13 & 4,4 & 6 & 2,0 & 3 & 1 \\
\hline Mayores de 45 años & 85 & 28,5 & 1 & 0,3 & 37 & 12,4 & 9 & 3,0 & 5 & 1,7 & 3 & 1 \\
\hline
\end{tabular}

hacer a $4 \mathrm{~mm}$ de la línea amelo-cementaría con el fin de estimar el hueso presente en la posición teórica ideal del implante dentro del alveolo. Tomando en consideración el valor de la mediana encontrado en el grupo 2 (mayores 45 años) se decide realizar un agrupamiento de los valores obtenidos en 6 subgrupos con intervalos de $0,50 \mathrm{~mm}$ y se realiza una nueva evaluación de los resultados mediante una prueba $\mathrm{X}^{2}$; obteniendo que en el grupo1 de mayores de 45 años el $60,7 \%$ de los cortes evaluados presentaban el valor 0 representando un $28,5 \%$ de la muestra total, a diferencia de los menores de 45 años donde el valor 0 se presentó solo en un 3,2\% del grupo lo cual equivale a un $1,7 \%$ de toda la muestra, adicionalmente en este grupo el grosor de hueso más frecuente se ubicó entre $1,00 \mathrm{~mm}$ a $1,50 \mathrm{~mm}$ lo que corresponde a un $82 \%$ del grupo, y a un $44,0 \%$ del total evaluado, estos valores fueron estadísticamente significativos. (Tabla 4)

\section{DISCUSIÓN}

La necesidad de una altura ósea de la pared vestibular suficiente para asegurar enmarcar el implante dentro de un hueso capaz no solo, de proporcionar estabilidad durante la inserción, sino también capaz de perdurar en el tiempo y evitando así problemas estéticos tales como: cambios de coloración en la mucosa o la visualización del implante, es una condición necesaria para alcanzar el éxito estético. El objetivo del presente estudio fue determinar las dimensiones promedio de altura y grosor de la cortical vestibular del maxilar anterior en una población de pacientes que acudieron a dos centros de Implantología de referencia. Estos valores podrían ser un factor relevante al momento de la planificación del implante inmediato, ya que pueden influir a la hora de elegir la longitud del implante, la profundidad de su colocación y la necesidad de regeneración ósea. Paralelamente se pretendía observar si factores como la edad, género o tipo de diente influyen de forma significativa en estas dimensiones.

Pocos estudios se han encontrado que evalúen altura de la cortical ósea vestibular maxilar como un factor predictivo para la implantología. Lin et al, al evaluar la altura ósea de 21 pacientes, con la finalidad de determinar la presencia de dehiscencias o fenestraciones y para así decidir el levantar un colgajo durante la colocación inmediata de implantes, encuentran una alta variabilidad de los valores con un rango de $2,79 \mathrm{~mm}$ a $11,29 \mathrm{~mm}^{(21)}$. Gracco et al, encontraron valores promedios de altura de $8,38 \mathrm{~mm}$ hasta $9,67 \mathrm{~mm}$ de altura de la cortical vestibular cuando evaluaron la posición y el soporte óseo del grupo incisivo en 191 pacientes $^{(22)}$. Estos valores, aunque menores, son similares a los observados en la presente investigación donde el promedio de altura ósea fue de 10,67 $\pm 1,46 \mathrm{~mm}$, con un rango de 4,00 hasta $13,50 \mathrm{~mm}$.

Acorde a lo esperado, el incisivo central presenta una mayor altura de hueso que el incisivo lateral, con $11,13 \mathrm{~mm}$ y $10,28 \mathrm{~mm}$ respectivamente, y aunque estas diferencias no resultan ser estadísticamente significativas, una diferencia aproximada de $1 \mathrm{~mm}$ podría implicar variaciones en la elección de la longitud del implante al momento de tratar estas zonas con implantación inmediata.

En la actualidad, la utilización de implantes con cambios de plataforma y las conexiones cónicas han sido consideradas como las más indicadas para el tratamiento de los sectores estéticos. Por ello se ha sugerido dejar el implante 1 a $2 \mathrm{~mm}$ subcrestal con la finalidad de prevenir la pérdida del hueso(13,18). Los resultados obtenidos, podrían sugerir que la longitud de implante más acertado a utilizar para tratar esta zona de forma inmediata a la extracción sería el de $13 \mathrm{~mm}$ de longitud, y este serviría tanto para el incisivo central como para el lateral, sin distingo entre edad o género, ya que no se aprecian diferencias significativas al comparar los grupos. No obstante, un detalle importante de resaltar es el hecho de que un $30,2 \%$ de los dientes evaluados no presentaron hueso a los $4 \mathrm{~mm}$ por debajo de la línea amelo cementaría.

Chappuis et al; comentan que los fenotipos periodontales delgados se asocian a un espesor de hueso cortical menor de $1 \mathrm{~mm}$, observándose una pérdida progresiva de la altura hasta de $7,5 \mathrm{~mm}$ luego de la implantación, lo cual sería incompatible con el éxito estético(23). Braut el al; encontraron un grosor menor de $1 \mathrm{~mm}$ en el más de $65 \%$ de 498 dientes evaluados a nivel vestibular y a nivel cervical(17). De igual forma, Nowzari et al, reportan que solo en un $3 \%$ de los pacientes se puede esperar un grosor mayor a 2 $\mathrm{mm}$ a $4 \mathrm{~mm}$ por debajo de la línea amelo-cementaría ${ }^{(24)}$. Estos resultados concuerdan con los obtenidos en la muestra donde el promedio de espesor de hueso cervical fue de $0,73 \pm 0,55$. no obstante son mayores a los obtenidos en la mayoría de las evaluaciones similares reportadas hasta la fecha ${ }^{(16,17,24,25)}$

Parece evidente, que un grosor superior a los $2 \mathrm{~mm}$ en el hueso crestal vestibular a nivel cervical, no es de esperarse en la mayoría de los pacientes, y este estudio lo confirma al observarse solo en 6 casos de toda la muestra evaluada, lo que equivale a un $2 \%$ de la muestra evaluada. Esto podría representar la necesidad de implementar técnicas de regeneración ósea para aumentar el volumen óseo y así poder evitar la pérdida del hueso crestal con el compromiso desde el punto de vista estético que esto ocasionaría.

Cabe señalar que hasta la fecha no se encontraron artículos que relacionen las dimensiones del hueso vestibular con el género o la edad del paciente. Diferencias significativas fueron observadas en cuanto al grosor observado entre géneros, donde las mujeres presentaron valores de 0,85 $\pm 0,04 \mathrm{~mm}$ comparados a los 0,59 $\pm 0,04$ observados en el género masculino. En una prueba piloto, realizada en la Facultad de Odontología de la Universidad de Carabobo, fue observada, una tendencia a perder las dimensiones del hueso crestal en pacientes de mayores de 45 años ${ }^{(20)}$. Los resultados observados en el presente estudio parecen confirman esta teoría, ya que una diferencia de más de 0,50 mm fue observada al comparar los grupos. No obstante, se deben realizar nuevas investigaciones encaminados a determinar el tipo de relación que existiría entre la edad y las dimensiones del hueso crestal tanto en altura como en grosor.

\section{CONCLUSIONES}

Dentro de las limitaciones que puedan derivarse de este estudio, se podría afirmar que en los incisivos maxilares la altura del hueso crestal vestibular parece ser suficiente para la colocación de implantes dentro de un marco de hueso. No obstante, un grosor menor a $1 \mathrm{~mm}$ debe esperarse en la mayoría de los casos, lo que podría traducirse en una necesidad de aplicar técnicas de regeneración tisular guiada, especialmente en situaciones de implantes inmediatos y en donde la estética sea un factor determinante.

\section{RELEVANCIA CLÍNICA}

Justificación del estudio: Las dimensiones del hueso alveolar son determinantes en la planificación y pronóstico de implantes inmediatos. Decisiones clínicas tales como, longitud del implante, regeneración ósea, y durabilidad de los resultados clínicos, dependen de una cresta óptima en términos de altura y grosor.

Resultados principales: La altura ósea observada parece ser suficiente para proteger al implante. No así el grosor óseo, el cual no es suficiente para evitar la pérdida de hueso posterior a la implantación, especialmente en pacientes mayores.

Consecuencias prácticas: La regeneración ósea y tisular deberán ser consideradas siempre como complemento de la implantación de este sector.

\section{CONFLICTO DE INTERÉS}

Los autores declaran no tener relación o conflicto de interés en este reporte. 


\section{Bibliografía}

1. Johnson K. A study of the dimensional changes occurring in the maxilla following closed face immediate denture treatment. Aust Dent J. 1969;14:370-376.

2. Lam RV. Effect of root implants on resorption of residual ridges. J Prosthet Dent. 1972;27:311-323.

3. Branemark PI, Adell R, Albrektsson T, Lekholm U, Lundkvist S, Rockler B Osseointegrated titanium fixtures in the treatment of edentulousness. Biomaterials. 1983;4:25-28

4. Chiapasco M, Gatti C, Rossi E, Haefliger W, Markwalder TH. Implant-retained mandibular overdentures with immediate loading. A retrospective multicenter study on 226 consecutive cases. Clin Oral Implants Res. 1997:8:48-57.

5. Chiapasco M, Gatti C. Implant-retained mandibular overdentures with immediate loading: a 3- to 8-year prospective study on 328 implants. Clin Implant Dent Relat Res. 2003;5:29-38.

6. Buser D, Chappuis V, Belser UC, Chen S. Implant placement post extraction in esthetic single tooth sites: when immediate, when early, when late? Periodontol 2000. 2017;73:84-102.

7. Chen ST, Buser D. Esthetic outcomes following immediate and early implan placement in the anterior maxilla--a systematic review. Int J Oral Maxillofac Implants. 2014;29 Suppl:186-215

8. Kan JY, Rungcharassaeng K, Lozada JL, Zimmerman G. Facial gingival tissue stability following immediate placement and provisionalization of maxillary anterio single implants: a 2- to 8-year follow-up. Int J Oral Maxillofac Implants. 2011;26:179-187. 9. Buser D, Martin W, Belser UC. Optimizing esthetics for implant restorations in the anterior maxilla: anatomic and surgical considerations. Int J Oral Maxillofac Implants 2004;19 Suppl:43-61.

10. AlKudmani H, Al Jasser R, Andreana S. Is bone graft or guided bone regeneration needed when placing immediate dental implants? A systematic review. Implant Dent. 2017;26(6):936-944.

11. Grunder U, Gracis S, Capelli M. Influence of the 3-D bone-to-implant relationship on esthetics. Int J Periodontics Restorative Dent. 2005;25:113-119.

12. Nevins $M$, Camelo $M$, De Paoli S, Friedland B, Schenk RK, Parma-Benfenati S, et al. A study of the fate of the buccal wall of extraction sockets of teeth with prominent roots. Int J Periodontics Restorative Dent. 2006;26:19-29.

13. Pellicer-Chover H, Diaz-Sanchez M, Soto-Penaloza D, Penarrocha-Diago MA Canullo L, Penarrocha-Oltra D. Impact of crestal and subcrestal implant placement upon changes in marginal peri-implant bone level. A systematic review. Med Oral Patol Ora Cir Bucal. 2019;24:e673-e683

14. Hammerle $\mathrm{CH}$, Chen ST, Wilson TG, Jr. Consensus statements and recommended clinical procedures regarding the placement of implants in extraction sockets. Int J Oral Maxillofac Implants. 2004;19(Suppl):26-28.

15. Kourtis S, Skondra E, Roussou I, Skondras EV. Presurgical planning in implant restorations: correct interpretation of cone-beam computed tomography for improved imaging. J Esthet Restor Dent. 2012;24:321-332.

16. Miyamoto Y, Obama T. Dental cone beam computed tomography analyses of postoperative labial bone thickness in maxillary anterior implants: comparing immediate and delayed implant placement. Int J Periodontics Restorative Dent. 2011;31:215-225. 17. Braut V, Bornstein MM, Belser U, Buser D. Thickness of the anterior maxillary facial bone wall-a retrospective radiographic study using cone beam computed tomography. Int J Periodontics Restorative Dent. 2011;31:125-131.

18. de Siqueira RAC, Savaget Goncalves Junior R, Dos Santos PGF, de Mattias Sartor IA, Wang HL, Fontao F. Effect of different implant placement depths on crestal bone levels and soft tissue behavior: A 5-year randomized clinical trial. Clin Oral Implants Res. 2020;31:282-293

19. Vandenbroucke JP, von Elm E, Altman DG, Gotzsche PC, Mulrow CD, Pocock SJ, et al. Strengthening the Reporting of Observational Studies in Epidemiology (STROBE) explanation and elaboration. Int J Surg. 2014;12:1500-1524

20. Parra E, Perenguez H, Labrador M, Sierra-Rebolledo A. Dimensiones óseas en la cortical vestibular maxilar del sector anterior, la edad y la condición sistémica de paciente. Departamento de Desarrollo Integral del Hombre. Valencia: University of Carabobo, 2013:61.

21. Lin CY, Pan WL, Wang HL. Facial fenestration and dehiscence defects associated with immediate implant placement without flap elevation in anterior maxillary ridge: a preliminary cone beam computed tomography study. Int J Oral Maxillofac Implants. 2018:33:1112-1118

22. Gracco A, Lombardo L, Mancuso G, Gravina V, Siciliani G. Upper incisor position and bony support in untreated patients as seen on CBCT. Angle Orthod. 2009;79:692702.

23. Chappuis V, Araujo MG, Buser D. Clinical relevance of dimensional bone and soft tissue alterations post-extraction in esthetic sites. Periodontol 2000. 2017;73:73-83.

24. Nowzari H, Molayem S, Chiu CH, Rich SK. Cone beam computed tomographic measurement of maxillary central incisors to determine prevalence of facial alveolar bone width $>/=2 \mathrm{~mm}$. Clin Implant Dent Relat Res. 2012;14:595-602.

25. Ghassemian M, Nowzari $H$, Lajolo C, Verdugo F, Pirronti T, D'Addona A. The thickness of facial alveolar bone overlying healthy maxillary anterior teeth. J Periodontol. 2012;83:187-197. 